\title{
Rapid Generation of $C^{2}$ Continuous Blending Surfaces
}

\author{
Jian J Zhang and Lihua You \\ National Centre for Computer Animation, Bournemouth University, \\ Poole BH12 5BB, United Kingdom
}

\begin{abstract}
Most surface-blending methods are able to blend surfaces with tangent continuity. However, curvature continuity has become increasingly important in geometric modelling and its applications, such as computer animation, computer-aided design and virtual reality. In this paper, we present a method which is able to achieve $C^{2}$ continuity based on the use of partial differential equations (PDE). A sixth order partial differential equation with one vector-valued parameter is introduced to produce such blending surfaces. Since computational efficiency is crucial for interactive computer graphics applications, we have developed a unified closed form (analytical) method for the resolution of this sixth order PDE. Therefore blending surfaces of up to $C^{2}$ smoothness can be generated in real time.
\end{abstract}

Key Words. surface blending, $C^{2}$ continuity, sixth order partial differential equation, closed form solution

\section{Introduction}

Surface blending is an important topic of surface modelling. There has been a great deal of effort devoted to this topic leading to the development of a number of surface blending methods. A comprehensive survey on existing blending methods for parametric surfaces was made by Vida et al. [20].

The rolling-ball blends, probably the most popular and classic surface blending method, generate blending surfaces with a moving ball. Depending on whether the radius of the ball changes or not, such blending method is classified as constant-radius blending [18], [10], [4] and variable-radius blending [11], [12], [16]. The main advantage of rolling-ball blending is that, since it is defined by a simple physical motion, the shape is generated in an intuitive manner. It is also attractive from the modelling point of view that the spine, the trimlines, the assignment and the profile are automatically generated. However, the surface swept by the moving ball is of a high algebraic degree, even in relatively simple cases.

Blends with cyclides were also investigated by some researchers [1], [2], [3], [19]. Cyclides can be regarded as generalisations of the torus and can be described by implicit quartic equations or in parametric form using trigonometrical parameterisation or rational biquadratic Bézier equations. When used for blending, the required cyclide pieces can be easily constructed by identifying circles on them, especially if these lie on the planes of symmetry. Cyclides are normally used in simple blends, such as where a cylinder obliquely meets a plane. 
Surface blending with the solution to a partial differential equation is also an effective method. This method was first proposed by Bloor and Wilson [5]. In the reported literature, however, the PDE based approach has only been used to construct a $C^{1}$ continuous blending surface. Another challenge is how to solve a PDE efficiently without compromising the geometric accuracy. Due to the difficulty of obtaining an analytical solution, various numerical methods are developed and used as mainstream solutions, which are inevitably very slow. Cheng [9] proposed a finite difference method and carried out the blending between two cylinders, and between a cylinder and a cone. Li et al. developed a boundary penalty finite element method for surface blending [13], [14], [15]. In addition to these, collocation method [6] and finite element method [8] were developed for free form surface generation. Finite difference method was also applied to solve dynamic PDEs for cloth simulation [21], [24]. Although they are effective in finding the solutions to the PDEs, solving a large set of linear algebra equations is generally very time-consuming. As a result, these numerical methods are unsuitable for the graphics applications requiring high computational efficiency. In order to generate surfaces faster, Bloor and Wilson developed a pseudospectral method [7]. However, this method has been found not accurate enough for certain applications [27].

The vector-valued parameter in a PDE has a strong influence on the shape of the generated blending surfaces. In order to provide designers with more shape control options, You and Zhang proposed a more general fourth order partial differential equation. It has three vector-valued parameters and covers all existing fourth order partial differential equations used for surface generation [22], [26]. Zhang and You also discussed the impact of the orders of the PDEs used for free form surface creation [25].

All the above-mentioned partial differential equations are of the fourth order which can only cope with positional and tangential boundary conditions. Therefore, they can only guarantee tangential continuity. In practical engineering design, however, blending surfaces with curvature continuity are required in many situations. For example, high-speed cams without curvature continuity can cause harmful impact. Due to the importance of this issue, the curvature continuity of blending surfaces has also attracted substantial attention. Pegna and Wolter [17] presented the Linkage Curve theorem for the design of curvature continuous blending surfaces. However, since the blending with curvature continuity is much more difficult to achieve than that with tangential continuity, rapid generation of curvature continuous blending surfaces remains an open research issue.

Blending based on PDEs has unique advantages over the conventional approaches, because curvature continuity can be readily incorporated into the boundary conditions of the PDEs. In this paper, we will introduce a sixth order partial differential equation to accommodate the requirement of $C^{2}$ continuity. Unlike other (numerical) resolution methods reported in the literature, we will develop a unified closed form solution (analytical solution) to the PDE, which is able to produce a blending surface interactively. 


\section{Partial Differential Equation and Closed Form Solution}

It is known that a fourth order partial differential equation can meet both positional and tangential boundary conditions. To satisfy the curvature boundary conditions, a sixth order PDE has to be used. Using the operator defined in (3), the following sixth order partial differential equation in a vector form can be applied for this purpose

$$
\frac{\partial^{6} \mathbf{x}}{\partial u^{6}}+3 \mathbf{a}^{2} \frac{\partial^{6} \mathbf{x}}{\partial u^{4} \partial v^{2}}+3 \mathbf{a}^{4} \frac{\partial^{6} \mathbf{x}}{\partial u^{2} \partial v^{4}}+\mathbf{a}^{6} \frac{\partial^{6} \mathbf{x}}{\partial v^{6}}=\mathbf{0}
$$

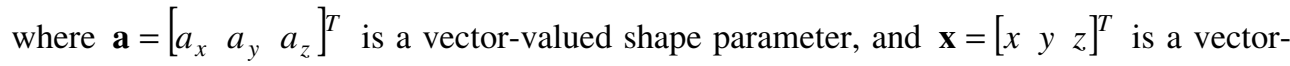
form positional function.

An arbitrary 3D surface can be represented with the solution to PDE (1) subject to position, tangent and curvature boundary conditions:

$$
\begin{array}{llll}
u=0 & \mathbf{x}=\mathbf{G}_{1}(v) & \frac{\partial \mathbf{x}}{\partial u}=\mathbf{G}_{2}(v) & \frac{\partial^{2} \mathbf{x}}{\partial u^{2}}=\mathbf{G}_{3}(v) \\
u=1 & \mathbf{x}=\mathbf{G}_{4}(v) & \frac{\partial \mathbf{x}}{\partial u}=\mathbf{G}_{5}(v) & \frac{\partial^{2} \mathbf{x}}{\partial u^{2}}=\mathbf{G}_{6}(v)
\end{array}
$$

where $\mathbf{G}_{i}(v)(i=1,2, \cdots, 6)$ represent the vector-valued functions which describes the position, tangent and curvature on the boundaries.

The blending surface generated with the solution to PDE (1) subject to boundary conditions (2) will guarantee curvature continuity. In fact, boundary conditions (2) are stronger than those of curvature continuity. Therefore, we here call the blending surfaces defined with boundary conditions (2) $C^{2}$ continuous blending surfaces.

PDE (1) under boundary conditions (2) can be solved with various numerical methods, such as the above mentioned finite element method, finite difference method, and collocation method as well as the weighted residual method [23]. Considering the importance of computational efficiency in interactive computer graphics, we will in this paper develop a unified closed form solution of PDE (1).

To facilitate the description, let us define a vector operator whose two operands are two column vectors, which produces a new column vector whose each element is the product of the corresponding elements of the two column vectors, i.e.

$$
\mathbf{s t}=\left[\begin{array}{lll}
s_{x} & s_{y} & s_{z}
\end{array}\right]^{T}\left[\begin{array}{lll}
t_{x} & t_{y} & t_{z}
\end{array}\right]^{T}=\left[\begin{array}{llll}
s_{x} t_{x} & s_{y} t_{y} & s_{z} t_{z}
\end{array}\right]^{T}
$$

Then decomposing the functions (2) into basic functions which are not in a polynomial form, boundary conditions (2) can be rewritten as follows

$$
\begin{array}{llll}
u=0 & \mathbf{x}=\sum_{i=1}^{I} \mathbf{a}_{1 i} \mathbf{g}_{i}(v) & \frac{\partial \mathbf{x}}{\partial u}=\sum_{i=1}^{I} \mathbf{a}_{2 i} \mathbf{g}_{i}(v) & \frac{\partial^{2} \mathbf{x}}{\partial u^{2}}=\sum_{i=1}^{I} \mathbf{a}_{3 i} \mathbf{g}_{i}(v) \\
u=1 & \mathbf{x}=\sum_{i=1}^{I} \mathbf{a}_{4 i} \mathbf{g}_{i}(v) & \frac{\partial \mathbf{x}}{\partial u}=\sum_{i=1}^{I} \mathbf{a}_{5 i} \mathbf{g}_{i}(v) & \frac{\partial^{2} \mathbf{x}}{\partial u^{2}}=\sum_{i=1}^{I} \mathbf{a}_{6 i} \mathbf{g}_{i}(v)
\end{array}
$$


where $\mathbf{a}_{j i}(j=1,2, \cdots, 6 ; i=1,2, \cdots, I)$ are known vector-valued coefficients, and $\mathbf{g}_{i}(v)(i=1,2, \cdots, I)$ are basic functions in a vector form.

Assuming that all $\mathbf{g}_{i}(v)(i=1,2, \cdots, I)$ can be expressed in the following forms (if some $\mathbf{g}_{i}(v)$ cannot be expressed in this way, they can be transformed into Fourier series to satisfy the requirements), then we have

$$
\begin{aligned}
& \mathbf{g}_{i}^{(2)}(v)=\mathbf{b}_{2 i} \mathbf{g}_{i}(v) \\
& \mathbf{g}_{i}^{(4)}(v)=\mathbf{b}_{4 i} \mathbf{g}_{i}(v) \\
& \mathbf{g}_{i}^{(6)}(v)=\mathbf{b}_{6 i} \mathbf{g}_{i}(v) \\
& (i=1,2, \cdots, I)
\end{aligned}
$$

where $\mathbf{g}_{i}^{(k)}(v)=\frac{d^{(k)} \mathbf{g}_{i}(v)}{d v}(k=2,4,6)$ and $\mathbf{b}_{k i}(k=2,4,6)$ are known vector-valued coefficients.

Corresponding to the basic functions, we can construct in the following a unified closed form solution of PDE (1) subject to boundary conditions (4) with the method of variable separation

$$
\mathbf{x}(u, v)=\sum_{i=1}^{I} \mathbf{f}_{i}(u) \mathbf{g}_{i}(v)
$$

Substituting (6) into PDE (1), the form of function $\mathbf{f}_{i}(u)$ can be determined. Depending on the values of $\mathbf{b}_{k i}(k=2,4,6), \mathbf{f}_{i}(u)$ has three different equations. Here we take the $x$ component as an example to present its equation.

If $b_{x 2 i}=b_{x 4 i}=b_{x 6 i}=0$,

$$
f_{x i}(u)=c_{x i 0}+c_{x i 1} u+c_{x i 2} u^{2}+c_{x i 3} u^{3}+c_{x i 4} u^{4}+c_{x i 5} u^{5}
$$

If $b_{x 2 i}=b_{x i}^{2}, b_{x 4 i}=b_{x i}^{4}, b_{x 6 i}=b_{x i}^{6}$,

$$
f_{x i}(u)=\left(c_{x i 0}+c_{x i 1} u+c_{x i 2} u^{2}\right) \cos a_{x} b_{x i} u+\left(c_{x i 3}+c_{x i 4} u+c_{x i 5} u^{2}\right) \sin a_{x} b_{x i} u
$$

And if $b_{x 2 i}=-b_{x i}^{2}, b_{x 4 i}=b_{x i}^{4}, b_{x 6 i}=-b_{x i}^{6}$,

$$
f_{x i}(u)=\left(c_{x i 0}+c_{x i 1} u+c_{x i 2} u^{2}\right) e^{a_{x} b_{x i} u}+\left(c_{x i 3}+c_{x i 4} u+c_{x i 5} u^{2}\right) e^{-a_{x} b_{x i} u}
$$

where $b_{x i}$ is the coefficient before the parametric variable $v$ in the basic functions $\mathbf{g}_{i}(v)$.

The unknown constants in equations. (7), (8) and (9) can be determined by substituting them into (6), then substituting (6) into boundary conditions (4).

\section{Application Examples}

In order to demonstrate the application of the above closed form solution in surface blending, in this section, we will create blending surfaces of three blending examples.

The first example is to blend an elliptic cylinder and a sphere which is used to explain the application of closed form solution (9). Suppose that the boundary conditions for this blending task have the forms of 


$$
\begin{aligned}
& u=0 \quad x=a \cos v \quad \frac{\partial x}{\partial u}=0 \quad \frac{\partial^{2} x}{\partial u^{2}}=0 \\
& y=b \sin v \quad \frac{\partial y}{\partial u}=0 \quad \frac{\partial^{2} y}{\partial u^{2}}=0 \\
& z=h_{0}-h_{1} \quad \frac{\partial z}{\partial u}=-h_{1} \quad \frac{\partial^{2} z}{\partial u^{2}}=0 \\
& u=1 \quad x=r \sin u_{0} \cos v \quad \frac{\partial x}{\partial u}=r \cos u_{0} \cos v \quad \frac{\partial^{2} x}{\partial u^{2}}=-r \sin u_{0} \cos v \\
& y=r \sin u_{0} \sin v \quad \frac{\partial y}{\partial u}=r \cos u_{0} \sin v \quad \frac{\partial^{2} y}{\partial u^{2}}=-r \sin u_{0} \sin v \\
& z=r \cos u_{0} \quad \frac{\partial z}{\partial u}=-r \sin u_{0} \quad \frac{\partial^{2} z}{\partial u^{2}}=-r \cos u_{0}
\end{aligned}
$$

Taking the $x$ component as an example and comparing (10) with (4) and (5), we have $g_{x 1}(v)=\cos v$ and $b_{x 21}=b_{x 61}=-1, b_{x 41}=1$. Therefore, the closed form solution of PDE (1) for the $x$ component is

$$
x=\left(\sum_{j=0}^{2} c_{x 1 j} u^{j} e^{a_{x} u}+\sum_{j=3}^{5} c_{x 1 j} u^{j-3} e^{-a_{x} u}\right) \cos v
$$

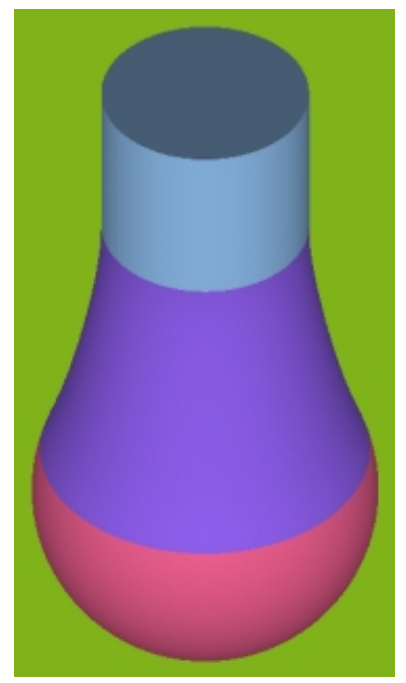

a

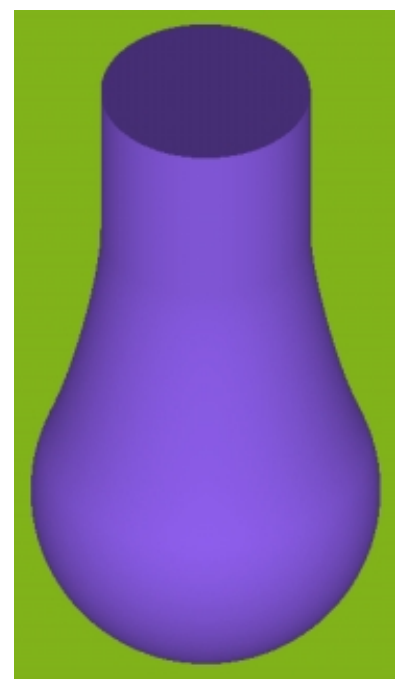

b

Fig. 1. Blending between an elliptic cylinder and a sphere

Substituting (11) into boundary conditions (10), all the unknown constants in (11) can be determined. With this closed form solution, we obtain the blending surfaces given in Fig. 1a and $1 \mathrm{~b}$. Figure 1a is the same as $1 \mathrm{~b}$ except that three different colours are used to distinguish 
the three surface patches. As the position, tangent and curvature continuities are all guaranteed at the boundary curves, as expected it looks very smooth (Figure 1b).

The second example is to blend a circular cylinder and a plane at a specified straight line. Its main aim is to demonstrate the application of the closed form solution (7) in surface blending. The boundary conditions for this blending problem are

$$
\begin{array}{rlrl}
u=0 & x=a_{0}-a_{1} v & \frac{\partial x}{\partial u}=2\left(a_{0}-a_{1} v\right) & \frac{\partial^{2} x}{\partial u^{2}}=2\left(a_{0}-a_{1} v\right) \\
y & =b_{0}+b_{1} v & \frac{\partial y}{\partial u}=2\left(b_{0}+b_{1} v\right) & \frac{\partial^{2} y}{\partial u^{2}}=2\left(b_{0}+b_{1} v\right) \\
z=0 & \frac{\partial z}{\partial u}=0 & \frac{\partial^{2} z}{\partial u^{2}}=0 \\
u=1 & x=r \cos v & \frac{\partial x}{\partial u}=0 & \frac{\partial^{2} x}{\partial u^{2}}=0 \\
y & =r \sin v & \frac{\partial y}{\partial u}=0 & \frac{\partial^{2} y}{\partial u^{2}}=0 \\
z & =h_{0}+h_{1} & \frac{\partial z}{\partial u}=h_{1} & \frac{\partial^{2} z}{\partial u^{2}}=0
\end{array}
$$

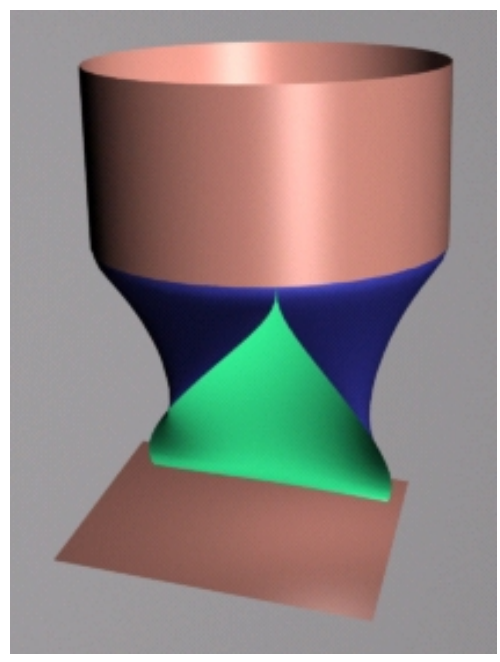

a

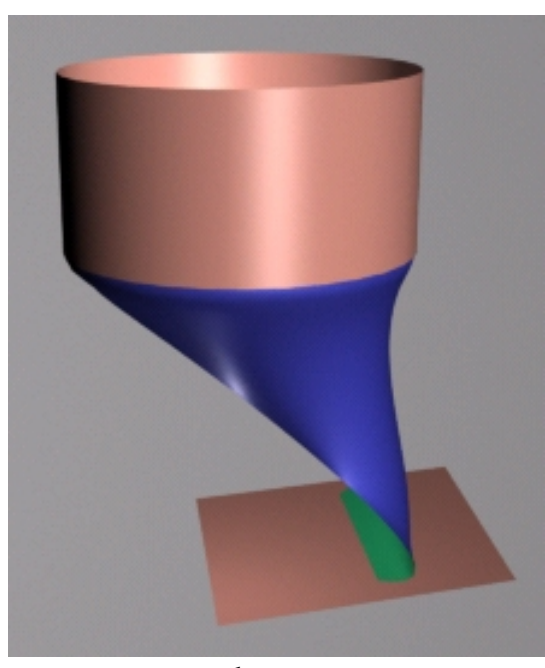

$\mathrm{b}$

Fig. 2. Blending between a circular cylinder and a plane at a specified straight line

For the $x$ component, we have $g_{1}(v)=1, g_{2}(v)=v$ and $g_{3}(v)=\cos v$. Therefore, the closed form solution of PDE (1) for the $x$ component takes the following form 


$$
x(u, v)=\sum_{j=0}^{5} c_{x 1 j} u^{j}+\sum_{j=0}^{5} c_{x 2 j} u^{j} v+\left(\sum_{j=0}^{2} c_{x 3 j} u^{j} e^{a_{x} u}+\sum_{j=3}^{5} c_{x 3 j} u^{j-3} e^{-a_{x} u}\right) \cos v
$$

In the same way as above, all the unknown constants in closed form solution (13) can be determined by substituting it into boundary conditions (12). The blending surface generated with (13) is depicted in Fig. 2a and 2b, which are from different viewing angles of the same blending surface.

The final example is to blend an elliptic cylinder and a plane at a specified curve. The boundary conditions for this blending task can be written as follows

$$
\begin{array}{rlrlrl}
u=0 & x=c \cosh d v & \frac{\partial x}{\partial u}=0 & & \frac{\partial^{2} x}{\partial u^{2}}=2 c \cosh d v \\
y & =c \sinh d v & \frac{\partial y}{\partial u} & =0 & \frac{\partial^{2} y}{\partial u^{2}}=2 c \sinh d v \\
z=0 & \frac{\partial z}{\partial u}=0 & \frac{\partial^{2} z}{\partial u^{2}}=0 \\
u=1 & x=a \cos v & \frac{\partial x}{\partial u}=0 & \frac{\partial^{2} x}{\partial u^{2}}=0 \\
y=b \sin v & \frac{\partial y}{\partial u}=0 & \frac{\partial^{2} y}{\partial u^{2}}=0 \\
z=h_{0} & \frac{\partial z}{\partial u}=2 h_{1} & \frac{\partial^{2} z}{\partial u^{2}}=2 h_{1}
\end{array}
$$

The basic functions for the $x$ component given by the above boundary conditions are $g_{x 1}(v)=\cosh d v$ and $g_{x 2}(v)=\cos v$. For $g_{x 1}(v), b_{x 21}=d^{2}, b_{x 41}=d^{4}, b_{x 61}=d^{6}$.

Therefore, the closed form solution of PDE (1) for the $x$ component is

$$
\begin{aligned}
x(u, v)= & \left(\sum_{j=0}^{2} c_{x 1 j} u^{j} \cos a_{x} d u+\sum_{j=3}^{5} c_{x 1 j} u^{j-3} \sin a_{x} d u\right) \cosh d v+\left(\sum_{j=0}^{2} c_{x 2 j} u^{j}\right. \\
& \left.e^{a_{x} u}+\sum_{j=3}^{5} c_{x 2 j} u^{j-3} e^{-a_{x} u}\right) \cos v
\end{aligned}
$$

Substituting (15) into boundary conditions (14) and determining all its unknown constants, we obtain the blending surface shown in Figure $3 \mathrm{a}$ and $3 \mathrm{~b}$. These two images are the same blending surface viewed from different viewing angles.

The last two examples are complex. The blending surface connects a closed curve of a circular/elliptic cylinder to an open curve (line/curve segment). The blending surface constructed using the partial differential equation smoothly merges from a closed shape at one end to an open shape at the other. We have not found similar blending surfaces in existing literature. They suggest that our method based on the solution of a PDE have ability to generate complex blending surfaces.

Because the solution is analytical, the developed closed form method is computationally very efficient. We have timed the process of determining the unknown constants in the 
closed form solutions and found the process took less than $10^{-6}$ of a second on a $800 \mathrm{MHz}$ PC for all three blending tasks, no problem for interactive computer graphics applications.

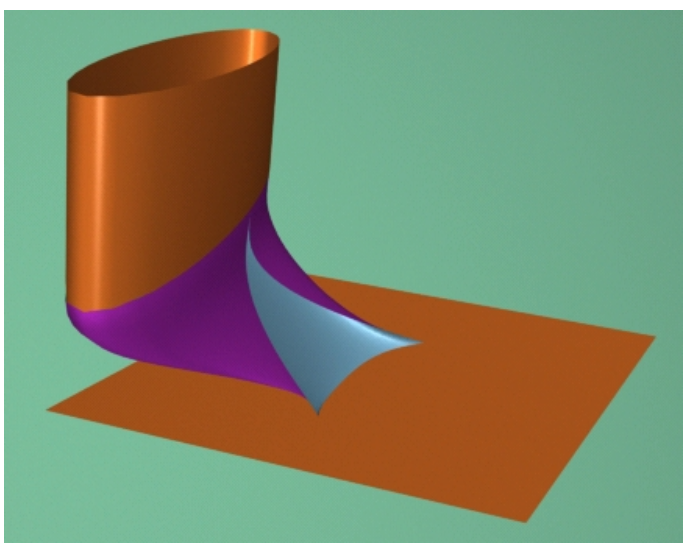

a

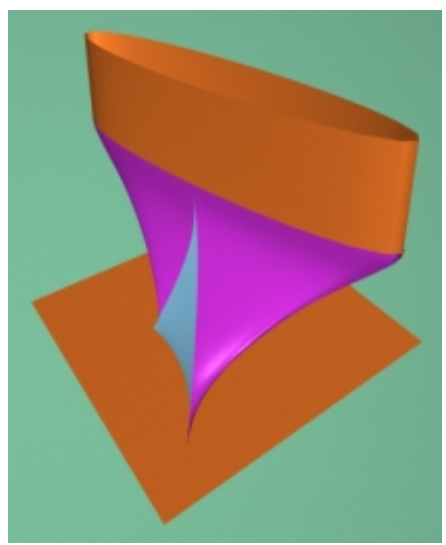

b

Fig. 3. Blending between an elliptic cylinder and a plane at a specified curve

\section{Conclusions}

Surface blending with $C^{2}$ continuity has been investigated in this paper. Different from the existing blending methods considering curvature continuity, we introduced a sixth order partial differential equation which provides enough degrees of freedom to accommodate curvature boundary conditions.

Since the computational speed is an important factor in interactive computer graphics applications, we developed a unified closed form resolution method. Depending on the differential properties of the boundary functions, the closed form solution of the PDE has three different forms.

By applying the developed closed form solution, we have given three examples to illustrate the use of our method. They are the $C^{2}$ continuous blending between an elliptic cylinder and a sphere, between a circular cylinder and a plane at a specified straight line, and between an elliptic cylinder and a plane at a given curve. These examples demonstrate the application of the three forms of the PDE solutions in surface blending (7), (8), (9), respectively. It is found that the developed blending method can tackle complex blending problems, some of which have not been seen in the literature. In addition, this method is computationally very fast, good enough for interactive applications. 


\section{References}

1. Allen, S., Dutta, D.: Cyclides in pure blending I, Computer Aided Geometric Design, 14 (1997) 51-75

2. Allen, G., Dutta, D.: Cyclides in pure blending II, Computer Aided Geometric Design, 14 (1997) 77-102

3. Allen, G., Dutta, D.: Supercyclides and blending, Computer Aided Geometric Design, 14 (1997) 637-651

4. Barnhill, R. E., Farin, G. E., Chen, Q.: Constant-radius blending of parametric surfaces, Computing Supple., 8 (1993) 1-20

5. Bloor, M. I. G., Wilson, M. J.: Generating blend surfaces using partial differential equations, Computer Aided Design, 21(3) (1989) 165-171

6. Bloor, M. I. G., Wilson, M. J.: Representing PDE surfaces in terms of B-splines, Computer Aided Design, 22(6) (1990) 324-331

7. Bloor, M. I. G., Wilson, M. J.: Spectral approximations to PDE surfaces, Computer Aided Design, 28(2) (1996) 145-152

8. Brown, J. M., Bloor, M. I. G., Bloor, M. S., Wilson, M. J.: The accuracy of B-spline finite element approximations to PDE surfaces, Computer methods in Applied Mechanics and Engineering, 158 (1998) 221-234

9. Cheng, S. Y., Bloor, M. I. G., Saia A., Wilson, M. J.: Blending between quadric surfaces using partial differential equations, in Ravani, B. (Ed.), Advances in Design Automation, Vol. 1, Computer Aided and Computational Design, ASME, (1990) 257-263

10. Choi, B. K., Ju, S. Y.: Constant-radius blending in surface modeling, Computer Aided Design, 21(4) (1989) 213-220

11. Chuang, J.-H., Lin, C.-H., Hwang, W.-C.: Variable-radius blending of parametric surfaces, The Visual Computer, 11 (1995) 513-525

12. Chuang, J.-H., Hwang, W.-C.: Variable-radius blending by constrained spine generation, The Visual Computer, 13 (1997) 316-329

13. Li, Z. C.: Boundary penalty finite element methods for blending surfaces, I. Basic theory, Journal of Computational Mathematics, 16 (1998) 457-480

14. Li, Z. C.: Boundary penalty finite element methods for blending surfaces, II. Biharmonic equations, Journal of Computational and Applied Mathematics, 110 (1999) 155-176

15. Li, Z. C., Chang, C.-S.: Boundary penalty finite element methods for blending surfaces, III, Superconvergence and stability and examples, Journal of Computational and Applied Mathematics, 110 (1999) 241-270

16. Lukács, G.: Differential geometry of $G^{1}$ variable radius rolling ball blend surfaces, Computer Aided Geometric Design, 15 (1998) 585-613

17. Pegna, J., Wolter, F.-E.: Geometrical criteria to guarantee curvature continuity of blend surfaces, Journal of Mechanical Design, Transactions of the ASME, 114 (1992) 201-210

18. Rossignac, J. R., Requicha, A. A. G.: Constant-radius blending in solid modeling, Computers in Mechanical Engineering, (1984) 65-73

19. Shene, C.-K.: Blending two cones with Dupin cyclides, Computer Aided Geometric Design, 15 (1998) 643-673

20. Vida, J., Martin, R. R., Varady, T.: A survey of blending methods that use parametric surfaces, Computer-Aided design, 26(5) (1994) 341-365

21. You, L. H., Zhang, J. J., Comninos P.: Cloth deformation modelling using a plate bending model, The 7th International Conference in Central Europe on Computer Graphics, Visualisation and Interactive Digital Media, (1999) 485-491 
22. You, L., Zhang, J. J.: Blending surface generation with a fourth order partial differential equation, The Sixth International Conference on Computer-Aided Design and Computer Graphics ( CAD/Graphics'99 ), Shanghai, China, 1-3 December, (1999) 1035-1039

23. You, L. H., Zhang, J. J., Comninos, P.: A solid volumetric model of muscle deformation for computer animation using the weighted residual method, Computer Methods in Applied Mechanics and Engineering, 190 (2000) 853-863

24. Zhang, J. J, You, L. H., Comninos P.: Computer simulation of flexible fabrics, The $17^{\text {th }}$ Eurographics UK Conference, (1999) 27-35

25. Zhang, J. J., You, L. H.: Surface representation using second, fourth and mixed order partial differential equations, International Conference on Shape Modelling and Applications, Genova, Italy, 7-11, May, (2001) 250-256

26. Zhang, J. J., You, L. H.: PDE based surface representation, Computers \& Graphics, 25(6) (2001), in print.

27. Zhang, J. J., You, L. H.: Surface blending using a power series solution to fourth order partial differential equations, Computer-Aided Design, under review, 2001 